\title{
New illumination technique for IR-video guided patch-clamp recording from neurons in slice cultures on biomembrane
}

\author{
Philippe Alix*, Jochen Winterer, Wolfgang Müller \\ Neuroscience Research Centre, Charité, Humboldt University Berlin, Schumannstrasse 20/21, D-10117 Berlin, Germany
}

Received 17 February 2003; received in revised form 9 April 2003; accepted 14 May 2003

\begin{abstract}
Slice cultures on biomembrane are the method of choice for studying $\mathrm{Ca}^{2+}$-dependent plastic changes occurring over several days to weeks. Using IR-differential interference contrast, good visualization of neurons in biomembrane slice cultures has been achieved despite a negative optical effect of the biomembrane, but epifluorescence imaging requires removal of a Wollaston prism and the analyzer. Here, we describe a novel illumination method to overcome this problem. Using optic fiber illumination at a shallow angle from the top of the slice culture, with or without additional illumination from the bottom, we obtained good cellular resolution of neurons in biomembrane slice cultures as well as in acute slices with an infrared-video camera. With this technique, we demonstrate visually guided whole-cell patch-clamp recording of $\mathrm{Na}^{+}-$and $\mathrm{K}^{+}$-currents as well as combination of whole-cell recording with fluorescence imaging of hippocampal and entorhinal cortex neurons in biomembrane slice cultures. Our inexpensive method should prove very useful for studying in vitro effects of long-term manipulations on membrane currents and intracellular Ca $\mathrm{Ca}^{2+}$-signaling. (C) 2003 Elsevier B.V. All rights reserved.
\end{abstract}

Keywords: Organotypic cultures; Biomembrane; Patch-clamp; Hippocampus; Entorhinal cortex; CA1 pyramidal neuron; IR-video microscopy

\section{Introduction}

Acute slices are very important tools for studying the physiological and pharmacological properties of central neurons and neuronal circuits. They have the main advantage that they retain the cytoarchitecture of the tissue of origin and give results largely in agreement with in vivo recording at much less technical effort. Slices give more stable recording conditions and allow pharmacological treatments by bath application. Therefore, slices are routinely used in numerous laboratories. Among the different techniques used to investigate these slices, patch-clamp is one of the most adapted to study individually one cell in its network, allowing direct interaction with the intracellular signaling pathways. While patch-clamp recordings can be obtained blind without visual control, infrared-video (IR-video) microscopy allows visual identification of the cell and there-

\footnotetext{
* Corresponding author. Tel.: +49-30-450-528203; fax: +49-30450-576904.

E-mail address: alix.philippe@caramail.com (P. Alix).
}

fore has become very popular. Image quality is enhanced either by differential interference contrast (DIC) or other special contrast enhancing illumination and image intensification video (Dodt and Zieglgänsberger, 1990; Dodt et al., 1999, 2002). The latter allows unimpeded epifluorescence measurements, as it does not use polarizers and Wollaston prisms in the epifluorescence pathway.

Unfortunately, acute slices survive in good condition only for several hours (around $8 \mathrm{~h}$ ) and therefore do not allow study of long-term processes, such as plasticity going on over days. Gähwiler has closed this time gap between in vivo and in vitro studies by developing a brain slice culture technique keeping CNS tissue alive for up to several weeks or even months and thus allowing long-term experiments (roller-tube cultures, for a review, see Gähwiler et al. (1997)). Regrettably, this technique is quite demanding and others had difficulties in obtaining good preparations.

Slice cultures became really popular with neuroscientists with the arrival of a much easier method developed by Stoppini et al. (1991), where slices are cultivated on a membrane and can be manipulated easily during several 
weeks in culture (Poulsen et al., 2002; Sato et al., 2002). In contrast to roller-tube cultures flattening to a quasimonolayer within a few days, membrane cultures retain a semi-three-dimensional structure (Gähwiler et al., 1997). Some blind patch and IR-DIC video guided patch-clamp recording in biomembrane slice culture have been reported (Pozzo-Miller et al., 1999; SpahrSchopfer et al., 2000; see also Bossu et al., 1996). An alternative IR-video illumination has been developed by Dodt et al. (1999, 2002). This type of illumination does not require DIC prism and analyzer behind the microscope objective and is therefore more convenient when combining patch-clamp recording with epifluorescence imaging. Good visualization of cells by this type of IRvideo guided microscopy is apparently obstructed, however, through optical disturbance by the membrane of either the illumination (upright microscope) or the observation optical path (inverted microscope).

In this study, we present a new, very affordable illumination system which is equally convenient for epifluorence imaging like the Dodt illumination. Our illumination has given us a good discrimination of CA1 pyramidal cells in hippocampal membrane slice cultures as well as entorhinal cortex layer II stellate neurons in combined entorhinal cortex-hippocampus slice cultures to routinely obtain patch-clamp whole-cell recordings from these cells.

\section{Methods}

For dissections and preparations of slice cultures utmost care was taken to maintain sterile conditions throughout the procedure. All solutions used were filtered sterile $(0.2 \mu \mathrm{m})$ and only opened in a sterile laminar flow hood.

\subsection{Preparation and culture of slices}

Combined entorhinal cortico-hippocampal slice cultures were prepared from 7- to 9-day-old Wistar rats and maintained as described before (Stoppini et al., 1991). Briefly, the animals were decapitated under deep ether anesthesia. Transverse $300 \mu \mathrm{m}$ thick slices were cut using a Vibratome (FTB Vibracut 2, FTB Feinwerktechnik, Bensheim Auerbach, Germany) in gassed $\left(95 \% \mathrm{O}_{2} / 5 \%\right.$ $\mathrm{CO}_{2}$ ) ice-cold artificial cerebrospinal fluid containing (in $\mathrm{mM}$ ): $129 \mathrm{NaCl}, 3 \mathrm{KCl}, 1.6 \mathrm{CaCl}_{2}, 1.8 \mathrm{MgCl}_{2}, 21$ $\mathrm{NaH}_{2} \mathrm{CO}_{3}, 1.25 \mathrm{NaH}_{2} \mathrm{PO}_{4}$ and 10 glucose (pH 7.4). The slices were transferred onto a biomembrane $(0.4 \mu \mathrm{m}$ Millicell culture plate inserts; Millipore, Eschborn, Germany) and incubated at the interface of a medium containing 50\% MEM, 25\% Hank's balanced salt solution (HBSS; Sigma, Deisenhofen, Germany), 25\% horse serum (Gibco), $2 \mathrm{mM}$ L-glutamine, $\mathrm{pH} 7.35$ with $5 \% \mathrm{CO}_{2}$ at $37{ }^{\circ} \mathrm{C}$. The antibiotic-free culture medium was completely replaced after day 2 and thereafter twice a week.

\subsection{Electrophysiology}

After 1 week of culture, the biomembranes were cut around a slice culture with a pair of microscissors and the slice culture transferred to a petri dish where it was kept at submerged conditions by nylon strings crossing a u-shaped platinum holder. The petri dish was transferred to the microscope stage and the preparation was perfused with a saline containing (in $\mathrm{mM}$ ): $129 \mathrm{NaCl}, 3$ $\mathrm{KCl}, 4 \mathrm{CaCl}_{2}, 4 \mathrm{MgCl}_{2}, 21 \mathrm{NaH}_{2} \mathrm{CO}_{3}, 1.25 \mathrm{NaH}_{2} \mathrm{PO}_{4}, 1$ $\mathrm{LiCl}$ and 10 glucose $(\mathrm{pH} 7.4)$ gassed with $75 \% \mathrm{~N}_{2} / 20 \%$ $\mathrm{O}_{2} / 5 \% \mathrm{CO}_{2}$. CA1 cells were visualized using an upright fixed stage microscope (BX50WI, OLYMPUS), an IRvideo camera (IR CDD camera, SANYO VCB3572IRP, Sanyo Japan) and two optic fibers to illuminate the preparation. The IR photos of the cells were digitized, enhanced and displayed using a video frame grabber card with software (pci Grabber-4plus, Phytec, Mainz, Germany), and stored for reference on the harddisk of the PC. A first optic fiber was positioned underneath the petri dish at an angle of about $30^{\circ}$ and a second above the preparation at a similar angle than the first one. Best resolution of cells inside slice cultures was obtained with maximal proximity of the light guides to the preparation and therefore the top light guide was, after protection by a parafilm, even inserted into the saline inside the petri dish (see Fig. 1C). Patch-clamp electrodes were pulled from GB150F8P borosilicate glass (Science Products GmbH, Hofheim, Germany) with a two step vertical puller (PP-830, Narishige, Tokyo, Japan). Filled with the internal solution containing (in $\mathrm{mM}$ ): $115 \mathrm{KCl}, 1 \mathrm{LiCl}, 0.1 \mathrm{MgCl}_{2}, 3 \mathrm{ATP}-\mathrm{Mg}, 5$ Glutathion, 40 HEPES, titrated at $\mathrm{pH} 7.4$ with $\mathrm{KOH}$, patch-pipettes had resistances of 5-8 M 2 . Patch-clamp in the whole-cell configuration was used to record membrane currents of the CA1 cells in the voltage clamp mode with an EPC-7 patch-clamp amplifier (HEKA, Lambrecht/Pfalz, Germany). Currents were digitized and stored on a computer data acquisition system (interface NI PCl-1200, National Instruments, Austin, TX, data acquisition software Cell Works, npi, Tamm, Germany). Current subtractions were performed with IGORPRO 3.0 software (Wavemetrics, Lake Oswego, OR). The conductance-voltage relation data were fitted with Prism software (3.02, GRAPHPAD) using the Boltzmann equation (Hlubek and Cobbett, 1997):

$g / g_{\max }=1 /\left(1+\exp \left(-\left(V-V_{0.5}\right) / k\right)\right)$

All potassium currents were measured in the presence of $1 \mu \mathrm{M}$ tetrodotoxin (TTX) in order to block $\mathrm{Na}^{+}$currents. 


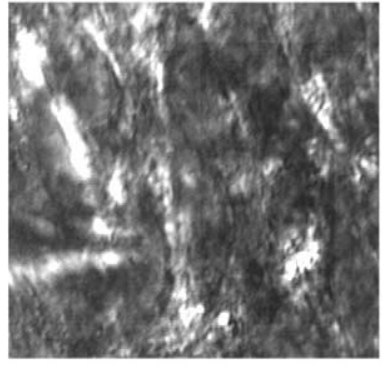

(a)

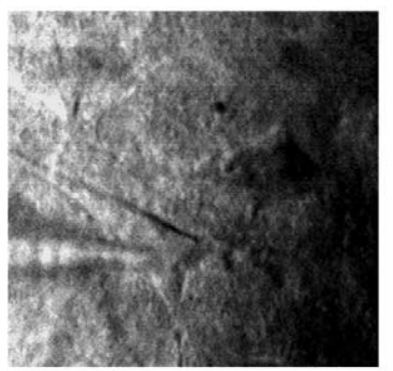

(b)

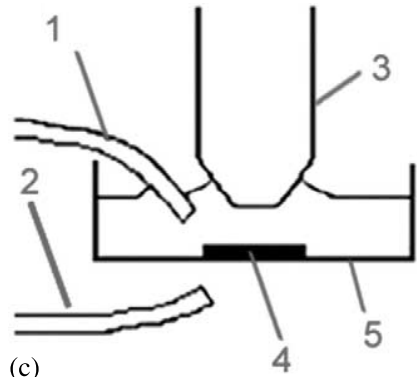

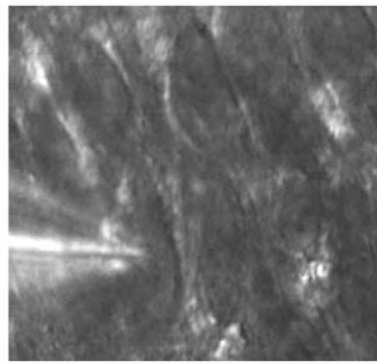
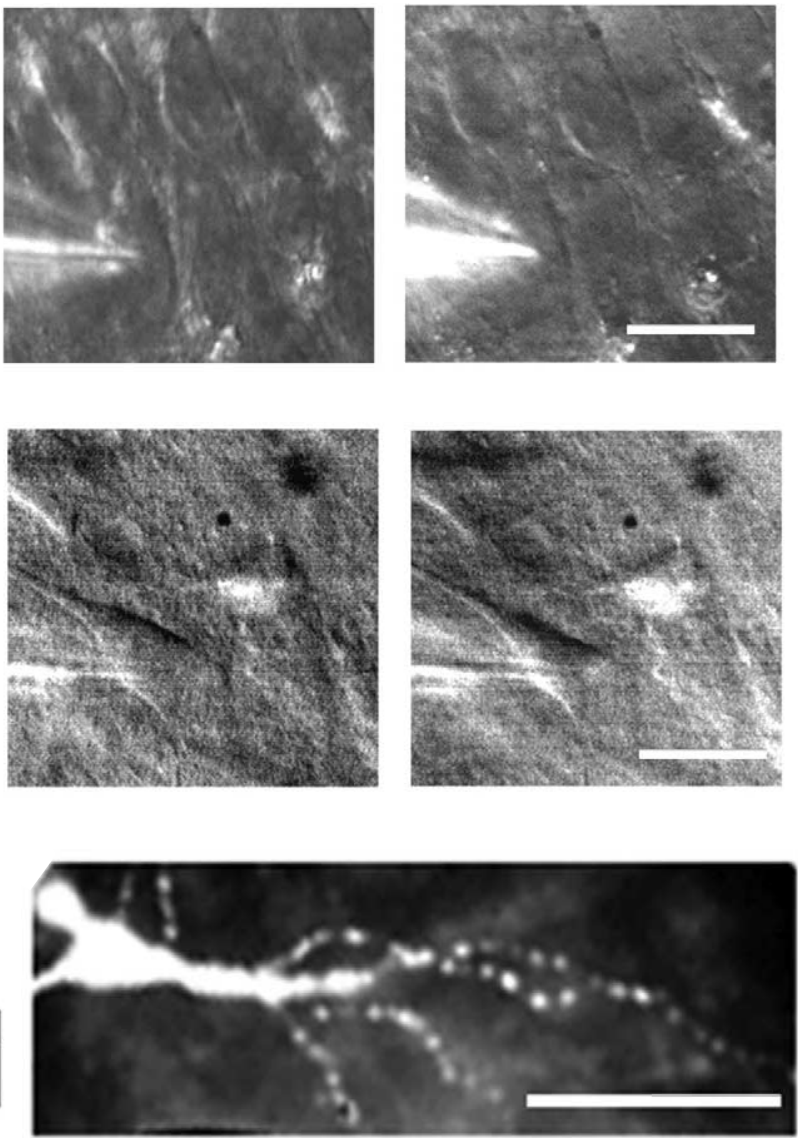

(d)

Fig. 1. IR-video microscopy with light guide illumination of acute slices and slice cultures for IR-video guided patch-clamp recording from neurons. (A) IR-video images of CA1 cells in membrane slice culture with the different ways of illuminations demonstrate an insufficient resolution for illumination only from underneath through the biomembrane (left) but a good cellular resolution with illumination from above (right), from above and from underneath (middle). Scale bar is $100 \mu \mathrm{m}$. (B) IR-video images of CAl cells in acute slice with the different illuminations show good resolution for illumination from underneath (left), combined from above and from underneath (middle), and from above (right; scale bar $100 \mu \mathrm{m}$ ). (C) Schematic representation of the microscope illumination setup used to patch cells inside CNS tissue ((1) optic fibers for the illumination of the preparation from above; (2) optic fibers for the illumination of the preparation from underneath; (3) water immersion objective of the microscope; (4) preparation; (5) recording chamber) (D) $\mathrm{Ca}^{2+}$-independent raw fluorescence image of a CA1 cell in membrane slice culture, whole-cell patch-clamp recording with FURA-2 $(200 \mu \mathrm{M})$ containing intracellular saline (excitation at $360 \mathrm{~nm}$; scale bar $100 \mu \mathrm{m})$.

\subsection{Fluorescence}

For fluorescence imaging, cells were patched with intracellular saline containing in addition $200 \mu \mathrm{M}$ of the membrane impermeable fluorescent $\mathrm{Ca}^{2+}$-indicator FURA-2 pentapotassium salt. For $\mathrm{Ca}^{2+}$-independent fluorescence morphology, an excitation wavelength of $360 \mathrm{~nm}$ was applied using a Polychrome II monochromator (TILL photonics, Gräfelfing, Germany) connected to the epifluorescence port of the microscope. Emission was observed using a 400-nm dichroic mirror, a 500-nm long path filter and a digital CCD camera (TE/CCD EFT back illuminated CCD, ST 138 Controller, Princeton Instruments, Princeton, NJ).

\section{Results}

\subsection{Patch-clamp set-up}

Following 1 week of culture, the viability of slice cultures has been checked by stimulating the connections between the CA1 region and the entorhinal cortex layer IV-VI with the paired pulse protocol and recording of postsynaptic field potentials (data not shown). We then tested our IR-video microscopy setups with acute slices positioned on lens cleaning paper underneath an upright fixed stage microscope. This test verified good optical resolution of individual CA1 pyramidal neurons in acute slice either with a Dodt illumination (Luigs \& 
Neumann, Ratingen, Germany; Leica upright microscope DMLFSA with a 1.4 oil condenser) or with a light guide (optic fibers bundle, KL 200, Schott, Mainz, Germany) at a shallow angle from underneath the preparation (Fig. 1C, Fig. 1B (left)). After this verification, we tested the IR-video microscopy resolution for organotypic slices cultivated on membranes. But, as illustrated in Fig. 1A (left), the use of a transillumination which was coming from below and crossing the biomembrane did not give sufficient resolution to obtain a clear discrimination of the cells, neither for the Dodt illumination (not shown) nor the light guide illumination (Fig. 1A (left)). To avoid the biomembrane being part of the optical path, we tested illuminations coming from above (Fig. 1C). The use of this light configuration gave a good visualization of CA1 pyramidal cells in slice culture (Fig. 1A (right)). The clearest sight of these cells was obtained by using both light sources in combination, illuminating from above and from underneath, simultaneously (Fig. 1A (middle)). The Fig. 1B (middle and right) illustrate, for comparison, the view of acute slice on lens cleaning paper, resulting from these last two lighting configurations, respectively.

\subsection{IR-video guided patch-clamp recording in membrane slice culture}

After selecting a neuron in the IR-video image with the combined illumination described in the previous section, the patch-pipette was lowered into the slice culture and brought into contact with the somatic membrane of the target neuron from the top. Fig. 1D demonstrates the compatibility of our IR-illumination method with epifluorescence microscopy, e.g. useful for $\mathrm{Ca}^{2+}$-imaging, by showing raw fluorescence of a Fura-2 filled CA1 pyramidal neuron at $360 \mathrm{~nm}$ excitation $\left(\mathrm{Ca}^{2+}\right.$-independent fluorescence). Whole-cell patchclamp recording of this CA1 neuron with intracellular saline containing $200 \mu \mathrm{M}$ Fura-2 resulted in a good filling of the cell and its dendritic tree within some 20 $\min$.

For electrophysiological analysis, cells were held at a potential of $-80 \mathrm{mV}$. By applying a voltage ramp

Fig. 2. Whole-cell patch-clamp recording of membrane currents in CA1 cells in membrane slice culture. (A) Voltage clamp recording of $\mathrm{Na}^{+}$-currents activated by a depolarizing ramp from -90 to $+10 \mathrm{mV}$. These currents resemble inverted action potentials, presumably due to limited space clamp and action potential like depolarizations in the dendritic tree. (B) Family of transient $\mathrm{K}^{+}$-current recordings $\left(I_{\mathrm{A}}\right)$ obtained by two different voltage protocols (see insert) and a subtraction procedure for isolating the A-current. Currents shown were activated by voltage steps to potentials between -50 and +10 $\mathrm{mV}$ in increments of $10 \mathrm{mV}$. (C) Current-to-voltage relationship of $I_{\mathrm{A}}$ (D) Voltage dependence of $I_{\mathrm{A}}$ conductance, normalized to maximal conductance. The line shows fit of the Boltzmann equation to the data.
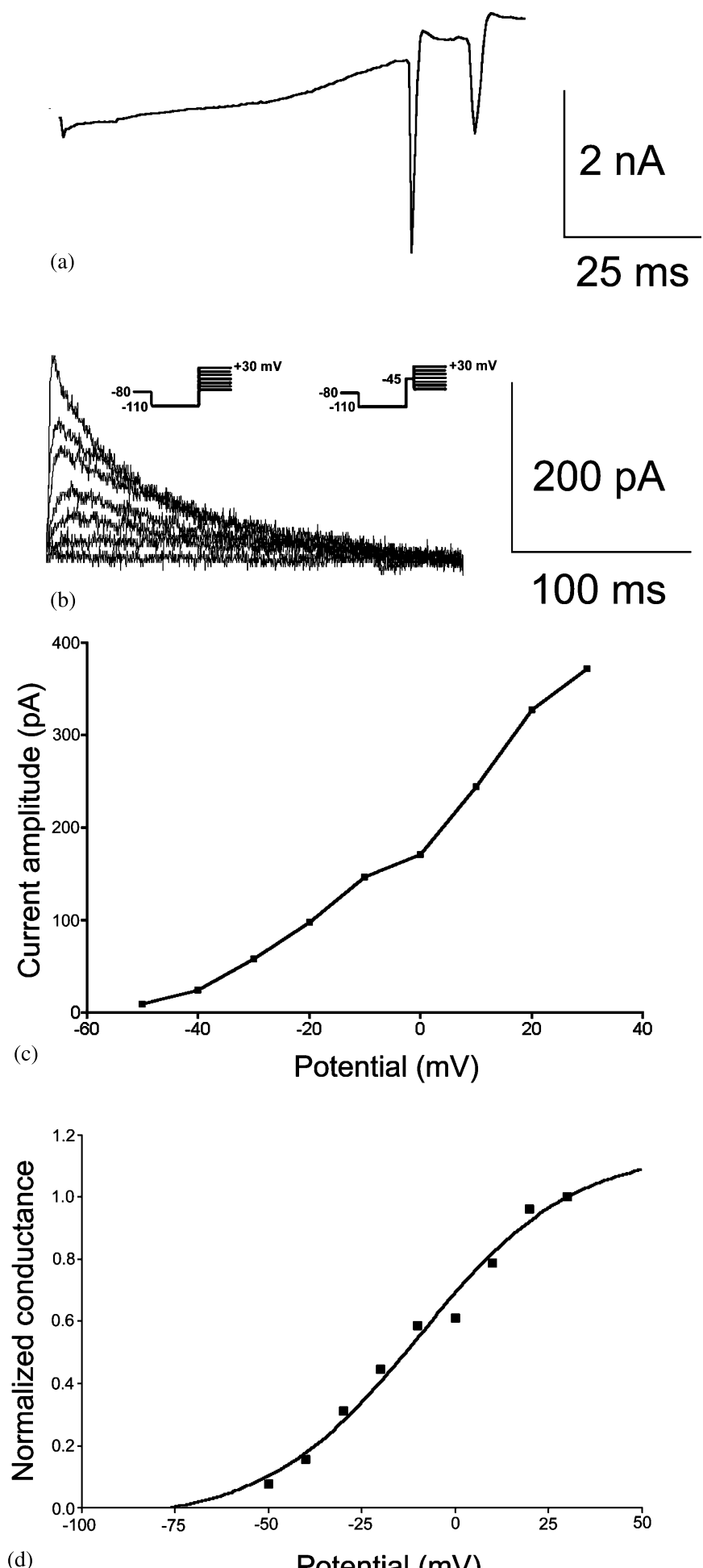

Potential $(\mathrm{mV})$

Fig. 2

protocol (pre step to $-90 \mathrm{mV}$ for $20 \mathrm{~ms}$, followed by a $150 \mathrm{~ms}$ ramp to $+10 \mathrm{mV}$ ), we tested for excitability of the recorded cell, i.e. the presence of strong voltageactivated $\mathrm{Na}^{+}$-currents $(n=4)$. These currents resembled inverted action potentials (Fig. 2A), presumably due to limited space clamp and action potential like 
depolarizations in the dendritic tree. Delayed rectifier $\left(I_{\mathrm{K}(\mathrm{V})}\right)$ and A currents $\left(I_{\mathrm{A}}\right)$ were recorded, during blockade of $\mathrm{Na}^{+}$-currents by TTX, using an $800-\mathrm{ms}$ hyperpolarizing prepulse to $-110 \mathrm{mV}$ to remove inactivation of $I_{\mathrm{A}}$. Hippocampal neurons $(n=5)$ were then step depolarized to potentials between -80 and + $30 \mathrm{mV}$ in increments of $10 \mathrm{mV}$. When the prepulse was followed by a $50-\mathrm{ms}$ interval at $-45 \mathrm{mV}, I_{\mathrm{A}}$ strongly inactivated, as has been described before (Connor and Stevens, 1971; Klee et al., 1995; Müller and Bittner, 2002). Although, unlike to the complete inactivation observed by these authors, inactivation of $I_{\mathrm{A}}$ was incomplete in our recordings. Nevertheless, by subtracting $I_{\mathrm{K}(\mathrm{V})}$ and the little part of $I_{\mathrm{A}}$ remaining from the mixed current with maximal $I_{\mathrm{A}}$, completely inactivating A-currents were obtained (Fig. 2B). One should be aware of that in this way $I_{\mathrm{A}}$ amplitudes have been somewhat underestimated. The voltage dependence of current activation is illustrated by the current-voltage curve in Fig. 2C. Fig. 2D shows the conductancevoltage relation for activation of $I_{\mathrm{A}}$ and the fit of the Boltzmann equation to these data.

\section{Discussion}

This new illumination method is extremely affordable and has been proven to allow efficient IR-video guided patch-clamp recording from neurons in membrane slice culture from 7- to 9-day-old rat, i.e. hippocampal CA1 pyramidal and entorhinal cortex layer II stellate neurons. In previous studies, patch-clamp whole-cell recordings have been performed on slice culture grown on biomembrane using the IR-DIC technique (PozzoMiller et al., 1999). Our method is significantly less expensive and is more convenient for combination with epifluorescence imaging. Thus, the possibility of easy patch-clamp recording with calcium imaging from cortical and hippocampal neurons in organotypic culture growing on biomembrane is a good augury for future studies of calcium dependent long-term plastic changes in intrinsic as well as excitatory and inhibitory synaptic membrane currents that underly physiological and pathophysiological processes.

Illumination from the bottom gives good resolution of neurons in acute brain slice, similar to illumination from the top. For slice cultures grown on biomembrane, apparently, illumination from the top side is essential for obtaining good optical resolution of individual neurons while additional illumination from below improves image quality at sites deeper within the tissue. In general, image quality improves with the proximity of the light guide to the preparation from the side at rather shallow angles. The light from the low-end models of socalled cold light sources is sufficiently bright for good illumination. Obviously, these light sources are intended for illumination at reduced heat load by reducing the emission of infrared light. Special coatings of the lamp reflector for emission of infrared light in the 800-1000 $\mathrm{nm}$ range might significantly enhance the light levels useful for IR-video microscopy. This would allow additional beam splitting for simultaneous observation of the preparation at two different optical magnifications.

Our illumination method does not interfere with epifluorescence methods and is much less expensive than IR-video microscopy based on DIC or Dodt illumination. Our light guide illumination has proven to be very useful in our laboratory for visually guided patch-clamp recording in acute slice as well as in biomembrane slice cultures. The latter is of particular importance, as we did not obtain using the Dodt illumination clear cellular resolution for visually guided patch-clamp recording in biomembrane slice culture. Moreover, our method is easy to employ and is not at all expensive. It offers the possibility to study effects of long-term treatment of developed neurons in tissue (cf. Poulsen et al., 2002; Sato et al., 2002) on their membrane properties and calcium signaling using the patch-clamp method.

\section{Acknowledgements}

The authors are grateful to Dr Oliver Kann, Sieglinde Latta and Dr Astrid Weber for expert advice and particularly to Pamela Liebig for her help in the preparation of the slice cultures.

\section{References}

Bossu J-L, Capogna M, Debanne D, McKinney RA, Gähwiler BH. Somatic voltage-gated potassium currents of rat hippocampal pyramidal cells in organotypic slice cultures. J Physiol 1996;495:367-81.

Connor JA, Stevens CF. Voltage clamp studies of a transient outward membrane current in gastropod neural somata. J Physiol 1971;213:21-30.

Dodt H-U, Zieglgänsberger W. Visualizing unstained neurons in living brain slices by infrared DIC-videomicroscopy. Brain Res 1990;537:333-6.

Dodt H-U, Eder M, Frick A, Zieglgänsberger W. Precisely localized LTD in the neocortex revealed by infrared-guided laser stimulation. Science 1999;286:110-3.

Dodt H-U, Eder M, Schierloh A, Zieglgänsberger W. Infrared-guided laser stimulation of neurons in brain slices. Science's STKE 2002;120:PL2.

Gähwiler BH, Capogna M, Debanne D, McKinney RA, Thompson SM. Organotypic slice cultures: a technique has come of age. Trends Neurosci 1997;20:471-7.

Hlubek MD, Cobbett P. Outward potassium currents of supraoptic magnocellular neurosecretory cells isolated from the adult guineapig. J Physiol 1997;502:61-74.

Klee R, Ficker E, Heinemann U. Comparison of voltage-dependent potassium currents in rat pyramidal neurons acutely isolated from 
hippocampal regions CA3 and CA1. J Neurophysiol 2000;74:1982-95.

Müller W, Bittner K. Differential oxidative modulation of voltagedependent $\mathrm{K}^{+}$currents in rat hippocampal neurons. J Neurophysiol 2002;87:29990-5.

Poulsen FR, Jahnsen H, Blaabjerg M, Zimmer J. Pilocarpine-induced seizure-like activity with increased BDNF and neuropeptide $\mathrm{Y}$ expression in organotypic hippocampal slice cultures. Brain Res 2002;950:103-18.

Pozzo-Miller LD, Inoue T, Murphy DD. Estradiol increases spine density and NMDA-dependent $\mathrm{Ca}^{2+}$ transients in spines of CA1 pyramidal neurons from hippocampal slices. J Neurophysiol 1999;81:1404-11.

Sato K, Matsuki N, Ohno Y, Nakazawa K. Effects of $17 \beta$-estradiol and xenoestrogens on the neuronal survival in an organotypic hippocampal culture. Neuroendocrinology 2002;76:223-34.

Spahr-Schopfer I, Vutskits L, Toni N, Buch P-A, Parisi L, Muller D. Differential neurotoxic effects of propofol on dissociated cortical cells and organotypic hippocampal cultures. Anesthesiology 2000;92:1408-17.

Stoppini L, Buchs PA, Muller D. A simple method for organotypic cultures of nervous tissue. J Neurosci Methods 1991;31:173-82. 